\title{
Bitter and sweet lupin (Lupinus albus L.) seeds and seed oils : a comparison study of their compositions and physicochemical properties
}

\begin{abstract}
In this study, bitter and sweet lupin (Lupinus albus L.) seed oils (BLO and SLO) were extracted using the soxhlet extraction method. The physicochemical properties, fatty acid compositions, thermal properties, 1H NMR, FTIR and UV visible spectra of BLO and SLO were evaluated. In addition, the antioxidant properties of bitter and sweet lupin seeds and their oils were also studied. The results showed that the bitter and sweet lupin seeds consist of $8 \%$ and $12 \%$ of oil, respectively. BLO and SLO contained high concentration of oleic acid (46.28 and 48.72\%), followed by linoleic acid (21.55 and 20.90\%), linolenic acid (7.69 and $8.95 \%$ ), and palmitic acid (7.39 and 7.5\%). The total tocopherol content of BLO and SLO were 184.70 and $317.01 \mathrm{mg} / 100 \mathrm{~g}$ oil, respectively. TG/DTG curves showed that the process thermal decomposition of the oils occurs in four phases for SLO and three phases for BLO.
\end{abstract}

Keyword: Lupin seed oil; Physicochemical properties; Antioxidant capacity; TGA/DTG; Cosmetic applications 